\title{
Developing architecture of a traveler information system for dynamic equilibrium in traffic networks
}

\author{
Mohammad Hossein Noranian ${ }^{1} \cdot$ Ahmad Reza Tahsiri $^{1}$
}

Received: 3 March 2017/Revised: 26 May 2017/Accepted: 27 May 2017/Published online: 6 June 2017

(c) The Author(s) 2017. This article is an open access publication

\begin{abstract}
Having criticized the current architecture of Advanced Traveler Information Systems (ATISs), this work discusses a new base of requirements to develop a new paradigm for traffic information systems. It mainly integrates three dimensions within a traffic system; drivers' pattern of behavior and preferences, urban traffic desires, and capabilities of traffic information service providers. Based on the above, functional segments from several related backgrounds are brought together to structure a new architecture, called Interactive Traveler Information System (ITIS). The main interactive feature of this new architecture is a two-way communication track between drivers and the traffic information system provider-in fact, a decision on choosing a road at a particular time for an individual will be made based on the utility of both sides. This new configuration consists of driver-side smartphone application, centric traffic prediction, and decision-maker units, which will shape a new approach of decision-making processes. These all work together to satisfy the designated goal of ITIS that it is preserving the Wardrop equilibrium condition in the traffic network level. Finally, we concentrate on a comparison study, which shows a differentiation between performance of the proposed ITIS and the current ATIS model in a real situation. This has been done with simulations of analogical scenarios. The most noticeable advantage of the proposed architecture is not being limited to a saturation limit, and
\end{abstract}

Mohammad Hossein Noranian

9124446@ee.kntu.ac.ir

Ahmad Reza Tahsiri

Tahsiri@kntu.ac.ir

1 Systems and Control Group, Electrical Engineering Department, KNT University of Technology, Tehran, Iran the positive effect of increasing system penetration in the performance of the newly introduced information system. In conclusion, new research subjects are suggested to be carried out.

Keywords Advanced Traveler Information System (ATIS) - System architecture - Requirement engineering · Mobile network · Dynamic equilibrium - Intelligent transportation system

\section{Introduction}

Advanced Traveler Information Systems (ATISs) are wellknown subsets of Intelligent Transportation Systems (ITSs) compared to other subsystems implemented until now. There are a lot of completely implemented ATIS projects in the USA and around the world, and their works are being discussed in the literature [1-6].

The first attempt at providing information and routing drivers was introduced in 1964 by General Motor's Driver Aid Information Routing (DAIR) system [7]. This system was one of the very first trials involving the use of different technologies to help drivers be informed and routed, while also being capable of asking for aid. This project was not successful because of a variety of equipment needed to be installed on the roads and, because of this, the tests were performed on the GM's test roads and never implemented on actual roads at all. In the 1970s, there were other regional projects introduced to the world, but the first commercial use of information systems was performed in Japan in 1986 with two projects-AMTICS and RACS - which were initially traveler information systems using car radios to broadcast the traffic information [8]. In 2000, the first nation-level project in traveler information system was launched: the 
number 511. The 511 telephone number is one of the earliest nationwide ATIS projects which are mostly widely known to the drivers [9]. After the improvements were implemented in the communication technologies and the wide access of civilians to internet and smartphones, newer players came to the competition. One of them was Google's Google Maps, which was launched in 2005, and in 2007, it began offering traffic data in real time. The data traffic collection source used by Google Maps is claimed to be a crowdsourcing model [10]. The Google Maps traffic service is accessible through the internet or the Google smartphone app.

The routing feature is one of the most interesting parts of traveler information system services. Based on the data provisioned by traffic information providers, numerous algorithms used by software applications and services were introduced that helped travelers find the best path for their trips [11-14]. In this context, "best path" may have many definitions. One of the first definitions for best path was shortest travel time. This understanding is the most common one for most people.

In the algorithms that find the shortest path and guide the drivers to the best path, the fact that this path is being suggested to all people who want to use that path is not noticed. It means that for a specific interval, based on penetration rate of available information system, a relatively large demand will be created for a path which is estimated to have the shortest travel time. But, because of the huge amount of demand generated for the path, it may change to the path with the longest travel time, because there is no control on the people making their decision based on the information gathered by them. These types of fluctuations may make traffic information system inefficient and may make traffic situation worse instead of making it better. Florian [15], based on a simulation scenario performed on an accepted simulation engine, showed that the best performance of current architecture of the ATIS system-which is based on blind and public dissemination of traffic information-is where just $30 \%$ of all the drivers use the benefit of the ATIS system and then, by the increase in the penetration percent of the ATIS system, travel time will increase too. This means that with a penetration rate higher than $30 \%$, increase in the users that use ATIS will cause longer travel times for both ATIS users and non-ATIS users.

In this paper, in the next section, the problems that current ATIS systems face will be stated and discussed. In the next section, by performing a requirement engineering process, a set of design goals will be extracted and the suggested ATIS architecture will be introduced based on the given design goals. The different subsystems of new architecture that is based on cellular communication network will be described in detail. At the end, the advantages and disadvantages of the suggested architecture will be stated with support of conceptual simulation results.

\section{Problem statement}

Before discussing the problems that the ATIS systems have faced, it's necessary to take a look to the current architecture of ATIS presented in Fig. 1. As shown in Fig. 1, the ATIS current architecture consists of a data gathering subsystem, data integration center, and traffic information dissemination infrastructure. Data gathering subsystems consist of cameras, aerial surveillance equipment, fixed sensors, and other available measuring tools. The data integration center is the place where all data are received and processed, and will be prepared as traffic information. The prepared information is then disseminated using available media such as television, variable signs, mobile phones, telephone, and internet. In advanced systems, the in-car monitoring devices will process the information provided by the ATIS system so that they can be used to find the shortest paths for travelers.

In Fig. 1, the broadcasting nature of the information system can be seen. This means that traffic information is being collected, integrated, and published in an iterative manner and it is intended to be applicable in drivers' decision-making processes and, hopefully, will make traffic situations easier on drivers. To better address the challenges of this architecture for traffic information systems, some references are reviewed. As mentioned above, Florian [15] indicated that this architecture for traffic information systems has a saturation threshold for the penetration rate of ATIS systems [15]. This phenomenon, called overreaction, is caused by the blind nature of the information dissemination and because there is no feedback on the usage of the information by drivers, the change in traffic patterns can't be predicted and controlled by the information disseminator [16]. This means that traffic information systems may make the traffic situation worse instead of better and that's not the whole story. Moreover, there are other scholars who challenged the current ATIS

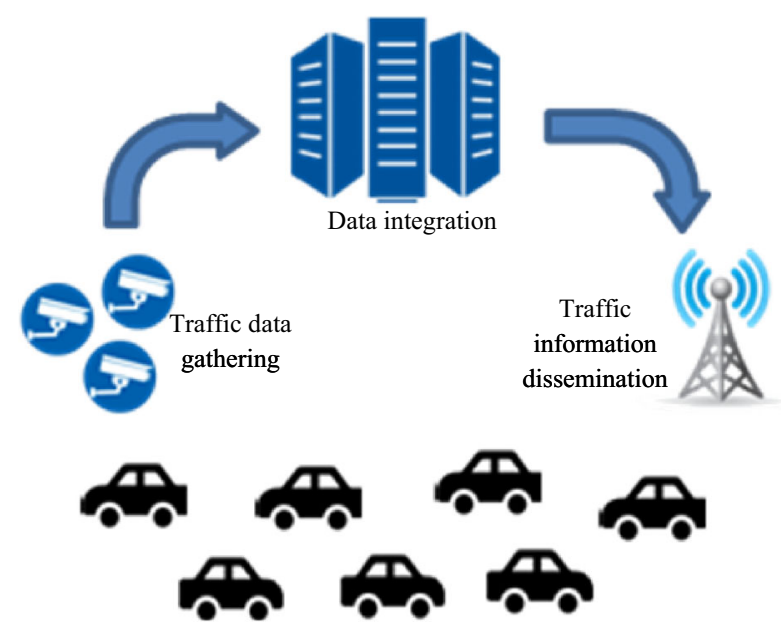

Fig. 1 Current architecture for ATIS 
system. In [17], some information dissemination mediums are being criticized and the current form of dissemination is questioned. The other wage dimension of the ATIS is its business model which needs to be revisited.

As pointed before, there are fundamental deficiencies which decay the operation of ATIS systems. They are listed below:

1. The reactive nature of traffic information can't be used when the traffic behaves dynamically. Travelers may choose a beaten path, but face busy paths because of traffic in links which enter the path, but the ATIS doesn't predict the traffic situation.

2. The public (blind) regime of information dissemination is another problem the current ATIS architecture faces. The effect of the public dissemination regime is fluctuations in traffic loading of different paths. This phenomenon is because of not considering other's decisions on traffic. If the traveler doesn't know the volume of demand for a path, his/her decision may not be the best choice at all.

3. For those who use raw traffic information, it's very hard for them to find the best path. Consider a lot of link segments with different colors, which present the level of service for each segment. Most of the system clients don't have necessary cognitive abilities to use this raw information, so it's not useful for most users.

4. Public information dissemination has no control benefit for the traffic control system. A costly process needs to be executed and an unknown number of users may use this system; the effect of this information dissemination is not precisely known. It may be waste of resources and sometimes causes unfavorable results.

On the other hand, dramatic increases in computational capabilities of processors, simple access to high-speed communication in cities and rural regions, and availability of smart phones for most citizens are new opportunities that ease the development of efficient interactive information systems. A variety of operating communication network companies are available that provide various services with relatively low prices. These features will be used in systems which will be developed hereafter. In the next section, a new architecture based on the technological advancements will be presented.

\section{Problem solution}

To find the best solution to the problem stated in the previous section, a suitable engineering suggestion is to execute the requirement engineering process for the ATIS and extract the design goals for the ATIS based on the requirements of the stakeholders of the system. As it can be seen, the major involved groups in the ATIS are drivers, traffic control organizations, and ATIS service providers. To perform the required engineering processes, requirements of each segment need to be indicated and processed in order to produce the design goals. The requirements of each of the stakeholders are listed and broken in hierarchical levels to cover more details of the requirement and, at the end stage, design goals are extracted by choosing unbreakable requirements in different levels. The list of requirements and different levels of work are presented in Table 1. As shown, some low-level requirements are being indicated many times; this highlights the significance of each. To lessen the complexity of the table, the duplicate low-level requirements are highlighted and lower levels are hidden.

To better clarify the requirement engineering process and to show how the design process is affected by design goals, a flowchart is depicted to show the process of system analysis and the design for the current case. As shown in Fig. 2, the requirement engineering process output is a design goal that will affect several parts of the system design flows, such as system process development, system architecture development, and subsystem feature design [18]. For this case, the requirements are affecting the system process block, in case the one-way flow of information is changed to a two-way flow between the information system center and the driver. The system architecture development block is affected by design goals to make it so that the cellular network is used and the server is distributed over a cloud-based hosting, which can better handle the huge load of computations during the traffic system simulation and decision-making process. The subsystem feature block is affected by using smartphones as an interface between the driver, the server, and the measuring node.The effects of the design goal on the system design flow are clearly described in Table 2 . In Table 2, the relation between the design goals and system features is clear and the relation between the design goals and system features is easily accessible. So, it's clear that each feature needs to meet specified requirements and the design process can be easier when more effective.

The new architecture for the ITIS is presented in Fig. 3. As shown in Fig. 3, the new architecture consists of a few extra parts, in addition to the current architecture, which makes core competencies for the new architecture. In the new architecture, which will be called Interactive Traveler Information System (ITIS), the most significant subsystems are Traffic Simulation Engine (TSE), Decision-Making Unit (DMU), Connection Front End (CFE), and Smartphone Application (SA). The communication infrastructure which will be used for communication between server and clients (drivers) is a cellular network that is a fully established and a fast improving network around the world. 
Table 1 Stakeholders' requirements list in hierarchical scheme

\begin{tabular}{|c|c|c|c|c|c|}
\hline & Requirements level 1: & & Requierments level 2 : & & Requirements level 3 : \\
\hline \multicolumn{6}{|l|}{ For drivers } \\
\hline DRL1-1 & Finding the path with the shortest travel time & DRL2-1 & Use of Traffic Assignment Algorithm & DRL3-1 & Individual driver oriented dynamic traffic assignment \\
\hline DRL1-2 & En-route path correction system & DRL2-2 & Enroute Traffic Assignment & DRL3-1 & Individual driver oriented dynamic traffic assignment \\
\hline \multirow[t]{3}{*}{ DRL1-3 } & low cost & DRL2-3 & Existing infrastructure can be used & DRL3-2 & Using smartphone over cellular communication \\
\hline & & DRL2-4 & Low energy consumption & DRL3-2 & Using smartphone over cellular communication \\
\hline & & DRL2-5 & Low communication cost & DRL3-2 & Using smartphone over cellular communication \\
\hline \multirow[t]{2}{*}{ DRL1-4 } & Customizable interface & DRL2-6 & Simple interface & DRL3-3 & Smartphone application \\
\hline & & DRL2-7 & Flexible interface & DRL3-3 & Smartphone application \\
\hline DRL1-5 & Protecting driver's privacy & DRL2-8 & Anonymous traffic assignment & DRL3-1 & Individual driver oriented dynamic traffic assignment \\
\hline \multirow[t]{2}{*}{ DRL1-6 } & Not to disrupt the driving functions & DRL2-9 & Disconnected to in-vehicle network & DRL3-3 & Smartphone application \\
\hline & & DRL2-10 & Not disturbing the driver & DRL3-3 & Smartphone application \\
\hline DRL1-7 & Noticing driver preferences & DRL2-11 & Considering driver decisions history & & \\
\hline DRL1-8 & Technical support availability & & & & \\
\hline \multicolumn{6}{|c|}{ For traffic control organization } \\
\hline ORL1-1 & Avoiding congestion for traffic network & DRL2-1 & Use of Traffic Assignment Algorithm & & \\
\hline ORL1-2 & Reliable travel time predictions & DRL2-1 & Use of Traffic Assignment Algorithm & & \\
\hline ORL1-3 & Encouraging for drivers & ORL2-1 & Can best fit the driver requirements & & \\
\hline \multirow[t]{2}{*}{ ORL1-4 } & Scalable & ORL2-2 & Most based on software capabilities & DRL3-3 & Smartphone application \\
\hline & & ORL2-3 & Shared resources can be used & ORL3-1 & Server over Cloud Architecture \\
\hline \multirow[t]{2}{*}{ ORL1-5 } & Low Cost & ORL2-4 & Low maintenance cost & ORL3-1 & Server over Cloud Architecture \\
\hline & & ORL2-5 & Low operating cost & ORL3-1 & Server over Cloud Architecture \\
\hline ORL1-6 & Applicable in traffic control & DRL2-1 & Use of Traffic Assignment Algorithm & & \\
\hline ORL1-7 & Fast implementation & DRL2-3 & Existing infrastructure can be used & & \\
\hline ORL1-8 & Useful for city announcements & ORL2-6 & Extended interface with upgradable & DRL3-3 & Smartphone application \\
\hline ORL1-9 & Reliability & ORL2-7 & Can be implemented with sufficient & ORL3-1 & Server over Cloud Architecture \\
\hline ORL1-10 & Security & ORL2-8 & $\begin{array}{l}\text { Data can be stored securely and } \\
\text { secure implementation can be done }\end{array}$ & ORL3-1 & Server over Cloud Architecture \\
\hline \multicolumn{6}{|c|}{ for ATIS Service Provider } \\
\hline SRL1-1 & Beneficial & SRL2-1 & Can charge at least a stakeholder for & & \\
\hline SRL1-2 & Fast Development and implementation & DRL2-3 & Existing infrastructure can be used & & \\
\hline SRL1-3 & Accessibility & ORL2-2 & Most based on software capabilities & & \\
\hline SRL1-4 & Reliability & ORL2-7 & can be implemented with sufficient & & \\
\hline SRL1-5 & Data can be usable for other purposes & SRL2-2 & $\begin{array}{l}\text { Data gathered can be stored and } \\
\text { Data Mining processes can be done }\end{array}$ & ORL3-1 & Server over Cloud Architecture \\
\hline \multirow[t]{2}{*}{ SRL1-6 } & Low necessary investments and costs & DRL2-3 & Existing platforms can be used & & \\
\hline & & ORL2-2 & Most based on software capabilities & & \\
\hline SRL1-7 & Robust to cyber-attacks and unexpected events & ORL2-8 & $\begin{array}{l}\text { Data can be stored securely and } \\
\text { secure implementation can be done }\end{array}$ & & \\
\hline SRL1-8 & $\begin{array}{l}\text { Availability of upstream service providers in a non- } \\
\text { exclusive market }\end{array}$ & DRL2-3 & Existing platforms can be used & & \\
\hline
\end{tabular}




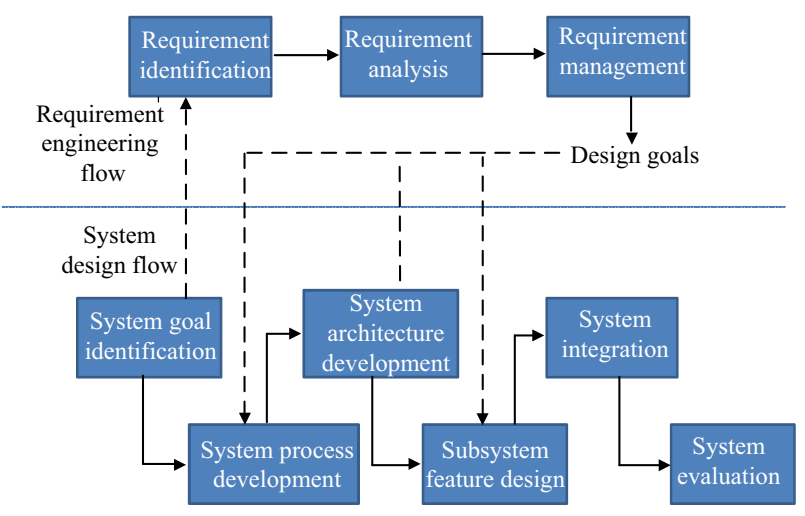

Fig. 2 Requirement engineering flow and system design flow and the interaction between the flows

Each section will be defined, and functions will be introduced. Each of the main sections is introduced in detail below:

Traffic Simulation Engine (TSE) All the information which is received from traffic control centers and drivers as vehicle probes will be integrated in this server to be used for predicting the traffic in the near future (about an hour). This place is called Traffic Simulation Engine (TSE) where the microscopic models are used and gathered data will be applied. In this architecture, the microscopic model used is Intelligent Driver Model (IDM), which was developed by Treiber et al. [19]. This model is a continuous model and computes acceleration of the vehicle by knowing the current velocity and headway of the preceding vehicle. This model also has six parameters that need to be calibrated. To calibrate the model for each car or for all the cars the same way, there is a managerial decision chosen by ITIS operators. Traffic Simulation Engine can predict the flow of each segment of the transportation network links. All the other active processes in the ITIS architecture use generated information.

To better show the trade-off between the advantages and disadvantages of car-following models used in the Traffic Simulation Engine (TSE), both the advantages and disadvantages are presented in Table 3 .

Decision-Making Unit (DMU) Decision-Making Unit (DMU) is a traffic assignment unit for vehicles which use ITIS. In other words, DMU decides each car that uses whichever path in an en-route strategy; whenever needed, the DMU changes the path of a vehicle and lets the vehicle know its best path considering the latest situation of the network traffic. This unit uses traffic assignment concepts to better assign the vehicles to the paths, and the criteria which are used to find the best choice are Wardrop Equilibrium criteria. In Wardrop Equilibrium, the traffic needs to be assigned in a way that no driver prefers to change his/her path because it makes no improvement in the experienced travel time.

Connection Front End (CFE) Connection Front End (CFE) is the interface between the drivers and server subsystems TSE and DMU. CFE will receive the position and behavior information of drivers from drivers (smartphone application in a driver's smartphone) and send the conditioned data to TSE. It also takes the latest decisions of the DMU (which are the latest paths for each driver) and send them to drivers. The importance of this interface is managing the communication tasks of the server, handling the communication related events, and handling the complexity of communicating with huge number of nodes connected to the server in real time.

The three units defined above are the server side units of the ITIS architecture. The subsystem of ITIS is a

Table 2 Design goal versus system features matrix

\begin{tabular}{|c|c|c|c|c|c|}
\hline \multirow[t]{2}{*}{ Design goals } & \multicolumn{5}{|c|}{ System features } \\
\hline & $\begin{array}{l}\text { Smartphone } \\
\text { application }\end{array}$ & $\begin{array}{l}\text { Cellular communication } \\
\text { network }\end{array}$ & $\begin{array}{l}\text { Traffic simulation } \\
\text { engine }\end{array}$ & $\begin{array}{l}\text { Decision- } \\
\text { making unit }\end{array}$ & $\begin{array}{l}\text { Connection } \\
\text { front-end }\end{array}$ \\
\hline $\begin{array}{l}\text { Individual based dynamic traffic } \\
\text { assignment }\end{array}$ & $\checkmark$ & & $\checkmark$ & $\checkmark$ & \\
\hline $\begin{array}{l}\text { Using smartphone over cellular } \\
\text { communication }\end{array}$ & $\checkmark$ & $\checkmark$ & & & \\
\hline Smartphone application & $\checkmark$ & & & & \\
\hline Server over Cloud Architecture & & $\boldsymbol{V}$ & & & $\boldsymbol{v}$ \\
\hline $\begin{array}{l}\text { Able to remember history of driver } \\
\text { decisions }\end{array}$ & $\checkmark$ & & $\checkmark$ & & $\checkmark$ \\
\hline $\begin{array}{l}\text { Able to charge at least a stakeholder } \\
\text { for the service }\end{array}$ & $\checkmark$ & $\checkmark$ & & & \\
\hline Technical support availability & $v$ & 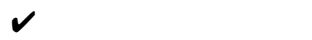 & & & $\checkmark$ \\
\hline
\end{tabular}




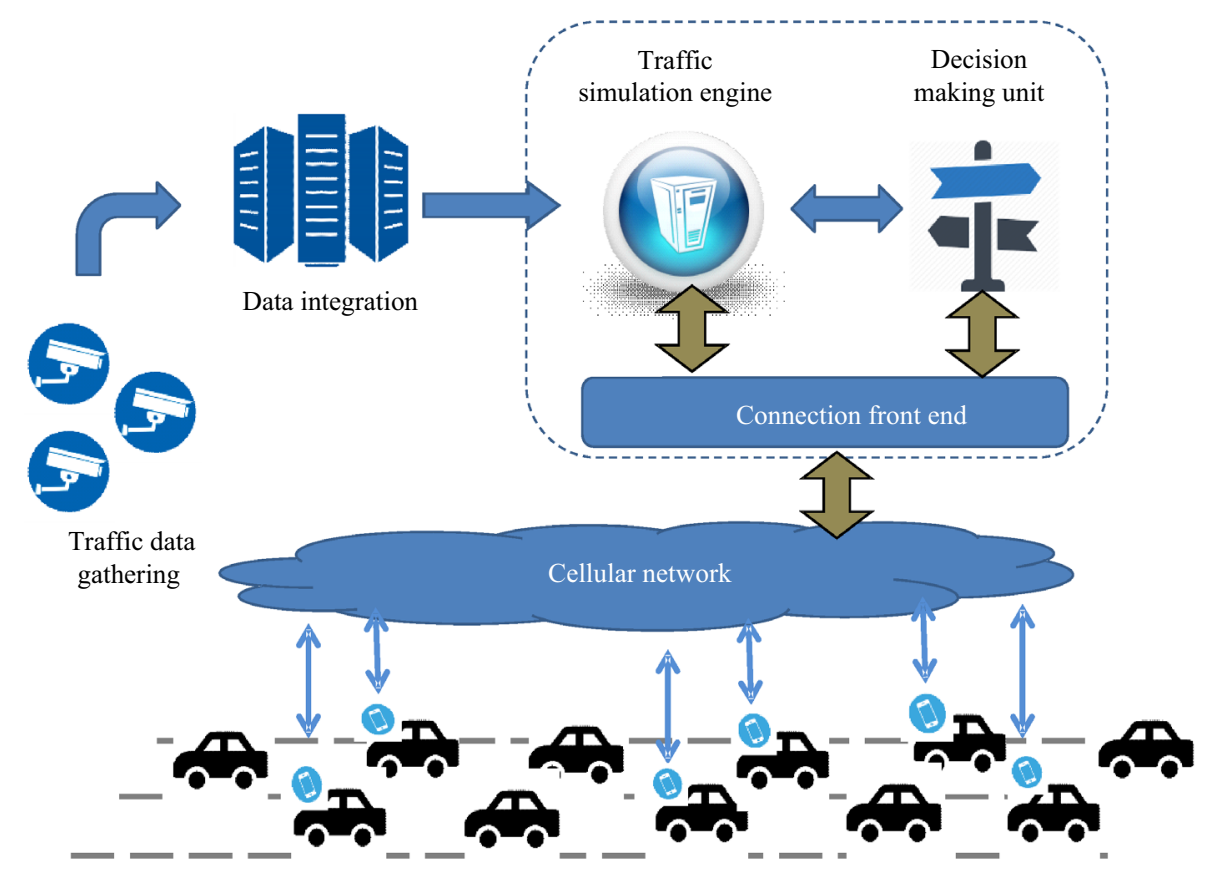

Fig. 3 Suggested architecture for ITIS

Table 3 Advantages and disadvantages of IDM in TSE

\begin{tabular}{ll}
\hline Advantages & Disadvantages \\
\hline High precision in modeling and predicting the traffic & Big computational load is created \\
Vehicles heterogeneity can be considered & Achieving information of all vehicles is required \\
Calculating the equilibrium criteria is more accurate & \\
Traffic predictions are more reliable &
\end{tabular}

smartphone application which will be introduced in the next paragraph.

Smartphone Application (SA) Smartphone Application (SA) is a mobile app which gathers the necessary information from vehicles; most smartphones are equipped with tools such as acceleration sensors, Global Positioning Systems (GPS), and cameras. It sends vehicle position and driver behavior parameters to the server through cellular communication networks and receives the recommended path from the server and informs the driver of the latest best path. The importance of SA in this architecture is that the spread of smartphones among drivers dramatically decreases the difficulty of equipping vehicles with devices having similar measurement capabilities. So, the ITIS architecture will be rapidly scalable and the implementation of such a system in a city can be very easy.

To better describe how this architecture works, the process of using this system is mentioned. At first, drivers send the destination to the server; this triggers DMU to assign a path to the driver from its origin to the specified destination. The vehicle's record in the server will be changed to active mode, and the vehicle will be considered in TSE as a moving vehicle. As the vehicle moves along the path, SA will send the position and behavior update of the vehicle to the server and TSE will use these data along with other data to better predict the network traffic. Based on predicted traffic, DMU will check the equilibrium criteria for the network and find gaps and change the path of vehicles in active decision mode; they can change their path because they entered splitting nodes in network. This leads to the situation of equilibrium state. This process continues until the vehicle reaches its destination.

One of the main improvements of ITIS architecture is the selective information dissemination which is implemented. This means the best path for each vehicle is being calculated and each path recommendation is considered a traffic situation, so the fluctuation problem that exists in public (blind) information dissemination doesn't repeat in the ITIS architecture; this makes it so that there is no saturation level for this system. Instead, the increase in the level of penetration of the ITIS will make it perform better 
and the level of disturbance in the estimation of traffic entered by non-equipped devices will be reduced.

Another main claim to the implementation of ITIS instead of current ATIS architecture is reaching the Wardrop equilibrium considering the heterogeneity in drivers' behavior. This means that all drivers will experience their best possible travel time. The equilibrium condition with Pareto optimality can be obtained whenever all the vehicles are equipped with the ITIS system. Also, even when just a portion of vehicles are equipped with this system, reaching a locally optimal equilibrium for the equipped vehicles is still possible. Equilibrium for equipped vehicles means that all the vehicles from a simultaneous OD pair can experience equal travel time, independent of their path; it's because the real-time evaluation of the traffic network helps find the best path for each equipped vehicle and lets the drivers be informed of their most efficient path, based on individual dynamic traffic assignment mechanisms. The definition of equilibrium is slightly different than the Wardrop equilibrium, but has the same level of optimality of the travel time, so it's applicable. In fact, for this form of equilibrium, non-equipped vehicles are considered disturbances, so in each step, by assigning the best path based on the available data to the equipped vehicles, a weaker sense of equilibrium will be achieved that is locally optimal. The en-route information dissemination feature helps drivers update their path with the updated traffic situation, caused by disturbance flows by unequipped vehicles.

To better depict the advantages and disadvantages of the presented architecture, they are being listed in Table 4. To clarify the impact of disadvantages presented in Table 4 , it can be said that computational power of available servers is relatively high and the problem of high-volume calculations is not serious in the current situation. The other problem of drivers with no smartphone is not inextricable too. Equipping drivers with a smartphone is much easier than equipping their vehicles with measurement devices

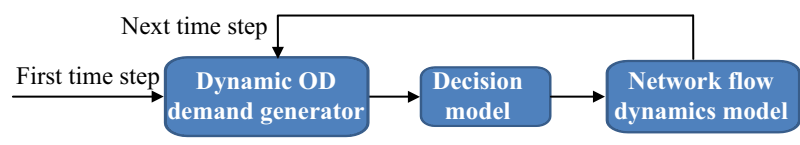

Fig. 4 Simulation process flow in each time step

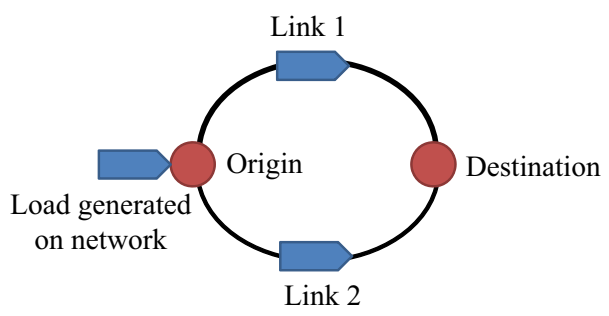

Fig. 5 First scenario network scheme

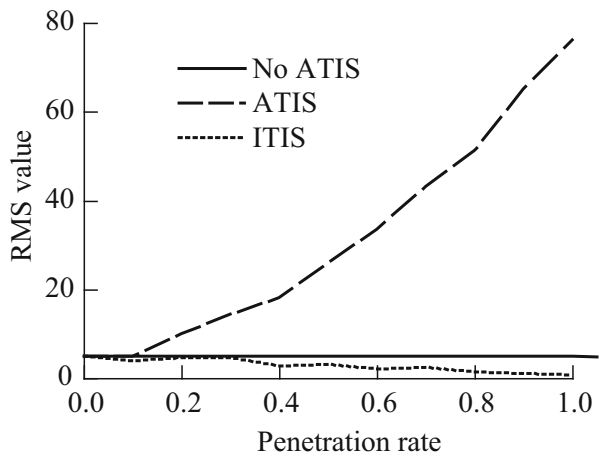

Fig. 6 RMS of travel time difference between two links in different information dissemination strategies for first scenario

both in availability and price. The challenge of drivers without smartphone will persist, and there is no solution for it but equipping them with a smartphone. Another challenge indicated in Table 4 is the heterogeneity of drivers' behavior. The drivers' heterogeneity is not modeled in a

Table 4 Advantages and disadvantages of ITIS architecture

\begin{tabular}{|c|c|}
\hline ITIS advantages & ITIS disadvantages \\
\hline Traffic situation will be estimated in the most precise way & $\begin{array}{l}\text { Huge amount of calculations are needed to implement the server } \\
\text { side }\end{array}$ \\
\hline $\begin{array}{l}\text { Recommending the best path to each driver will be possible in selective } \\
\text { manner }\end{array}$ & $\begin{array}{l}\text { If heterogeneity of drivers need to be considered, modeling } \\
\text { measure and traffic assignment algorithms need to be developed }\end{array}$ \\
\hline $\begin{array}{l}\text { Driver behavior can be used to calibrate the model of each driver in Traffic } \\
\text { Simulation Engine (TSE) }\end{array}$ & Some of the drivers may not have smartphones \\
\hline \multicolumn{2}{|l|}{$\begin{array}{l}\text { Traffic situation can be controlled based on the intelligent dissemination } \\
\text { regime }\end{array}$} \\
\hline \multicolumn{2}{|l|}{$\begin{array}{l}\text { Monitoring and guidance of the driver can be customized to best lead the } \\
\text { driver to the right path }\end{array}$} \\
\hline $\begin{array}{l}\text { Available infrastructure and devices are used to control the traffic that } \\
\text { results in rapid and efficient implementation of information system }\end{array}$ & \\
\hline
\end{tabular}




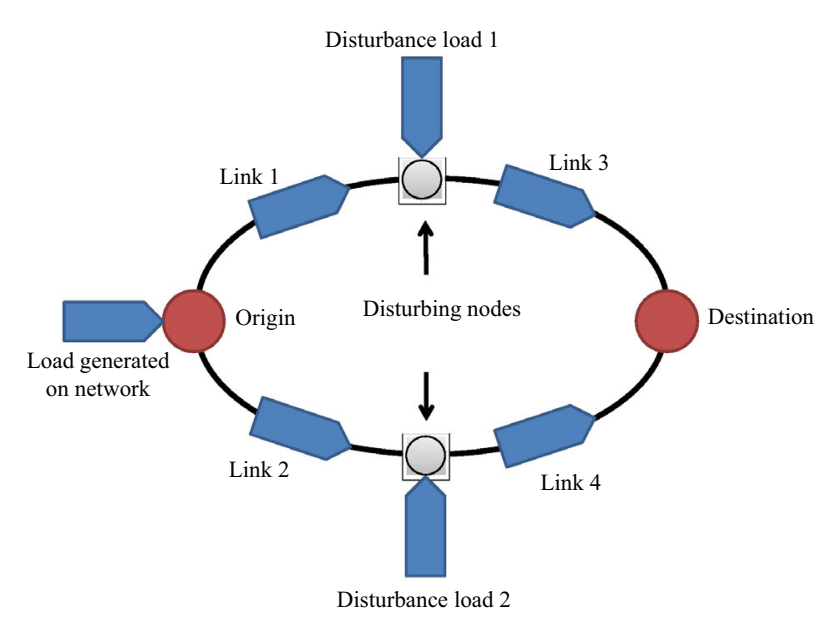

Fig. 7 Second scenario network scheme

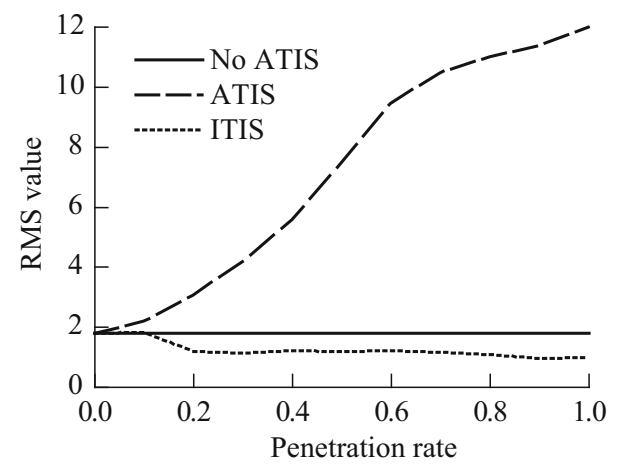

Fig. 8 RMS of travel time difference between two paths in different information dissemination strategies for second scenario

way can be used in the Traffic Simulation Engine (TSE). So this challenge leads to the development of models and algorithms consistent with heterogeneity.

To ensure the competency of new architecture and to improve the travel time reliability, in the next section, conceptual simulation results are provided and the results are discussed.

\section{Simulations}

The simulation results are provided to show how the selective information dissemination strategy can overcome the overreaction phenomena, which occurs in public information dissemination strategies that are used in current ATIS architectures. So, the main requirement that deals with this conceptual simulation is development of an individually based dynamic traffic assignment mechanism. In fact, the other requirements are implementation-level requirements that will be considered in the implementation phase of the subsystems. So, a simple working simulation scenario is presented to show the differences between the current and the newly introduced architecture for traveler information system. To better show how the architecture is utilized to perform the simulation, the simulation process scheme is depicted in Fig. 4.

As shown in Fig. 4, the simulation process consists of OD demand generation, decision modeling, and network flow dynamics model. The dynamic OD demand generator is a random value generator which generates the input volume for the network. The decision model, based on the information dissemination strategy, models the driver's decision-making process using ATIS and/or ITIS decisionmaking mechanisms and the logit model for non-equipped vehicles. The network flow dynamics model is the main segment of the simulation engine that simulates the movements of vehicles in the network based on car-following models (the IDM model in this case).

As indicated in the requirement engineering process, the different levels of requirements are presented hierarchically based on how requirements are transformed into design goals after the analysis phase. The design goals are what designers need to search for in the subsystems of the designed system. To do so, Table 2 clearly shows how the subsystems are customized in a way the design goals can be met. In the conceptual simulation process, which is shown in Fig. 4, the decision model is a down-scaled version of the decision-making unit in the main architecture, while the network flow dynamics model is a downscaled version of the traffic simulation engine in the main architecture. This means the conceptual simulation scenario is a down-scaled implementation of the real-scale subsystems in order to show the impact of new architecture of information system on the traffic situation.

In the first scenario, an origin-destination pair is assumed to be connected through the same links as depicted in Fig. 5.

Each link is one lane, each $500 \mathrm{~m}$ long. The maximum allowed speed for links is $10 \mathrm{~m} / \mathrm{s}$. To calculate the travel time for each link, the BPR model is being applied with $\alpha$ as 0.5 and $\beta$ as 4 . The free flow travel time and capacity of the road are being calculated using available equations for them in the references [20-22]. The load entered into the network is supposed to be uniformly random with maximum value of 20 vehicles per time interval. A unique load profile is applied in different simulation experiments to ensure the analysis of the results be reliable.

Three information dissemination strategies are being applied to the vehicles in the simulation. The first strategy is no information dissemination at all. In this case, because of the similarity of the roads, the vehicles choose one of the links and the load in them is the same with a bounded random variation. The second strategy is public information dissemination, which means the travel times of two 
links are being informed to vehicles equipped with the information system. In this case, different penetration percentages for information system are being supposed, so the simulation is being executed for different penetration rates. The third strategy is selective dissemination, which is the main idea of the new information system architecture introduced in this paper. The penetration rate of the information system in this strategy is being varied from $0 \%$ to $100 \%$ to find the impact on the system by increase in the penetration rates of the information system. In this paper, the penetration rate is defined as the operating portion of the equipped vehicles, which means the vehicles complying with the system are considered and vehicles not obeying the advice are not included in the operating penetration rate.

The simulation results are presented in Fig. 6. In Fig. 6, the Root Mean Square (RMS) for travel time difference in two links is presented for all the strategies introduced above and for different penetration rates. As shown in Fig. 6, the travel time difference in the first strategywithout information dissemination-is a specific value and the variation in different penetrations is because of the variation in random variables and there is no penetration at all. For the second strategy, the public dissemination strategy used in the current ATIS architecture, by increasing the penetration of the information dissemination system, the fluctuations caused by blind dissemination results in large differences between the links that is not acceptable. For the third strategy, which is based on selective dissemination, the RMS of travel time difference is converging to zero; that means the increase in penetration rates of this information dissemination system results in better travel time reliability and far better traffic situations.

In the second scenario, to show the impact of disturbances on the performance of the information dissemination strategies, the network is changed in order to include new merging nodes with disturbing loads, as shown in Fig. 7. There are four same links in the network with properties like the links in the first scenario. So, the link performance functions - which calculate travel time-will be the same. In the second network, there are two merging nodes with disturbing loads that disorder the total travel time of each path from origin to destination. By running the simulation for different information dissemination strategies, the effect of disturbing loads on the total performance of the strategies can be analyzed and the competency of the newly introduced information system architecture can be seen.

To do this, the disturbing load is random with one-tenth in amplitude, compared to the amplitude of the main load network. The penetration rate is calculated based on the number of vehicles equipped with the information system in the main network load stream, and the disturbing loads are not included in penetration calculation. The simulation results for all the three strategies are depicted in Fig. 8.

As shown in Fig. 8, the RMS value of travel time difference for the second strategy is ascending with the increase in penetration rate, which means that the increase in penetration rate will make the equilibrium condition worse. But the third strategy, which is the strategy suggested in this paper, is descending with the increase in penetration rate; this means an increase in the penetration of the ITIS system, even in the presence of disturbing loads, is working and making the traffic situation closer to equilibrium.

\section{Conclusion}

As indicated in this paper, the main contribution of the research is to find a way to solve the problems current ATIS are facing. To do so, a new architecture for the ATIS is introduced based on the requirements presented in the paper. The introduced architecture consists of different subsystems working together to provide the requirements of the different stakeholders of the system which are drivers, traffic control organizations, and ATIS service providers.

As stated in the advantages and disadvantages of the new architecture, the most important advantage of the architecture is scalability of the system, both in utilization and performance. This means that all the drivers can use the system and all drivers can benefit from the use of this system. On the other hand, the largest disadvantage of the architecture is the massive size of calculation tasks that compare terribly to the computational power of today's processors.

The effect of the suggested traffic information dissemination architecture is conceptually presented in the simulation results to show how it can make the travel time reliability better and how it affects the travel time of the links in the network.

Open Access This article is distributed under the terms of the Creative Commons Attribution 4.0 International License (http:// creativecommons.org/licenses/by/4.0/), which permits unrestricted use, distribution, and reproduction in any medium, provided you give appropriate credit to the original author(s) and the source, provide a link to the Creative Commons license, and indicate if changes were made.

\section{References}

1. Kumar P, Singh V, Reddy D (2005) Advanced traveler information system for Hyderabad City. IEEE Trans Intell Transp Syst 6(1):26-37 
2. Goetzenbrucker G, Koehl M (2012) Advanced traveller information systems for intelligent future mobility: the case of 'AnachB' in Vienna. IET Intel Transp Syst 6(4):494-501

3. Zhang X, Yang Z, Zhao S (2009) Research on ATIS information service media choice behavior of public transit traveler. In: International conference on management and service science, 2009. MASS'09, IEEE, pp 1-4

4. Yi Z, Xiaoguang Y, Meiping Y, Xuemei Z (2010) The relationship between urban travelers' personal attributes and propensity to utilize pre-trip traveler information system. In: 18th International conference on geoinformatics, 2010, IEEE, pp 1-5

5. Bar-Gera H (2007) Evaluation of a cellular phone-based system for measurements of traffic speeds and travel times: a case study from Israel. Transp Res Part C Emerg Technol 15(6):380-391

6. Alexandri D, Iordanopoulos P, Chrysostomou K, Mitsakis E (2015) Impacts of advanced traveler information systems: the case of the city of Patras. In: International conference on models and technologies for intelligent transportation systems (MT-ITS), 2015, IEEE, pp 265-272

7. Sussman JM (2005) Perspectives on intelligent transportation systems. Springer, Berlin

8. Miles JC (2014) Intelligent transport systems: overview and structure (History, Applications, and Architectures). Encycl Automo Eng 1-16

9. Stanley LM, Kelly MJ, Lassacher S (2005) Driver perfromance while interacting with the 511 travel information system in urban and rural traffic. In: Proceedings of the third international driving symposium on human factors in driving assessment, training and vehicle design, pp 486-492

10. Campbell PA, Havlicek JP, Stevenson AR, Barnes RD (2012) Realization of ITS applications through mapping technologies: a survey of advanced traveler information systems. In: 15th International IEEE conference on Intelligent transportation systems (ITSC), 2012, IEEE, pp 788-795

11. Bast H, Delling D, Goldberg A, Müller-Hannemann M, Pajor T, Sanders P, Wagner D, Werneck RF (2016) Route planning in transportation networks. In: Kliemann L, Sanders P (eds) Algorithm engineering. Springer, pp 19-80
12. Chen BY, Lam WH, Sumalee A, Li Q, Tam ML (2014) Reliable shortest path problems in stochastic time-dependent networks. J Intell Transp Syst 18(2):177-189

13. Toth P, Vigo D (eds) (2014) Vehicle routing: problems, methods, and applications. Society for industrial and applied mathematics, pp 193-212

14. Yao B, Hu P, Lu X, Gao J, Zhang M (2014) Transit network design based on travel time reliability. Transp Res Part C: Emerg Technol 43:233-248

15. Florian DG (2004) Simulation-based evaluation of advanced traveler information services (atis). Massachusetts Institute of Technology, Cambridge

16. Balakrishna R, Koutsopoulos H, Ben-Akiva M, Fernandez Ruiz B, Mehta M (2005) Simulation-based evaluation of advanced traveler information systems. Transp Res Rec J Transp Res Board 1910:90-98

17. Lyons G, Avineri E, Farag S, Herman R (2007) Strategic review of travel information research. Technical report. Department for transport, London. Available from: http://eprints.uwe.ac.uk/ 10300

18. Haskins C, Forsberg K, Krueger M (2010) INCOSE systems engineering handbook, vol 3. 1. International council on systems engineering, San Diego

19. Treiber M, Hennecke A, Helbing D (2000) Congested traffic states in empirical observations and microscopic simulations. Physical review E 62(2):1805

20. Moses R, Mtoi ET (2017) Calibration and evaluation of link congestion functions. J Transp Technol 4(2)

21. Mtoi ET, Moses R (2014) Calibration and evaluation of link congestion functions: applying intrinsic sensitivity of link speed as a practical consideration to heterogeneous facility types within urban network. J Transp Technol 4:141-149

22. Akcelik (1991) Travel time functions for transport planning purposes: Davidson's function, its time dependent form and alternative travel time function. Aust Road Res 21(3):49-59 